\title{
Perinatal Outcome and Change in Body Mass Index in Mothers of Dichorionic Twins: A Longitudinal Cohort Study
}

\author{
Teresinha Simões,' Alexandra Cordeiro,' Catarina Júlio,' José Reis,' Elsa Dias,' and Isaac Blickstein² \\ ' Department of Maternal-fetal Medicine and Neonatology, Maternidade Dr Alfredo da Costa, Lisbon, Portugal \\ ${ }^{2}$ Obstetrics and Gynecology, Kaplan Medical Center, Rehovot, Israel, and the Hadassah-Hebrew University School of Medicine, Jerusalem, Israel
}

W e used a prospective cohort to analyze the effect of change in BMI rather than change in weight, in mothers carrying dichorionic twins from a population that did not receive any dietary intervention. A total of 269 mothers (150 nulliparas and 119 multiparas) were evaluated. The average change (\%) from the pre-gravid BMI was $7.2 \pm 6.1,17.4 \pm 8.2$, and $28.7 \pm 10.8$, at 12-14, 22-25, and 30-34 weeks, respectively, without difference between nulliparas and multiparas. The comparison between maternities below or above the average change from the pregravid BMI failed to demonstrate an advantage (in terms of total twin birthweight and gestational age) of an above average change from the pregravid $B M I$, even when the lower versus upper quartiles were compared. Our observations reached different conclusions regarding the recommended universal dietary intervention in twin gestations. A cautious approach is advocated towards seemingly harmless excess weight gain, as normal weight women may turn overweight, or even obese, by the end of pregnancy, and be exposed to the untoward effects of obesity on future health and body image.

Preterm birth and low birthweight are the most significant complications of twin gestations. In the United States, more than one of every four very low birthweight (VLBW) infants $(<1500 \mathrm{~g})$ comes from a multiple birth (Martin et al., 2005), and nearly one of every five deaths within the first month of birth were born in a multiple delivery (MacDorman et al., 2005). Specifically, the 2002 clinical statistics from the United States suggest that as many as $58.2 \%$ and $11.9 \%$ of twins are born preterm $(<37$ weeks) and very preterm (<32 weeks), respectively, and as many as $55.4 \%$ and $10.2 \%$, respectively, are low $(<2500 \mathrm{~g})$ or very low $(<1500 \mathrm{~g})$ birthweight infants. (Martin et al., 2003) These figures suggest that more risky groups of infants - those delivered very preterm and/or with a VLBW - are roughly 7 to 9 times more prevalent among twin than among singleton gestations (Martin et al., 2003). The significant contribution of twins to overall preterm and low birthweight rates is further emphasized by the fact that birth rates of twins are still increasing, as opposed to the stabilized or even decreasing birth rates of higher-order multiples (Blickstein \& Keith, 2005).

Regrettably, there are no practical means to reduce these adverse outcomes of twin pregnancies to the comparable singleton levels. Indeed, it seems unrealistic to expect that twin births would have similar outcomes to singleton births. A more realistic approach would be to focus on methods that may reduce the more risky subgroup of twins, namely to reduce the rates of very preterm and VLBW infants. In this respect, the seminal work of Luke and her coworkers seems to be of utmost importance. In both retrospective and prospective cohorts, Luke and her colleagues observed a significant increase in birthweight and gestational age in twins whose mother gained enough weight during early (up to 24 weeks) pregnancy. (Luke et al., 1991; Luke et al., 1993; Luke \& Leurgans, 1996; Luke et al., 1997; Luke, 1998; Luke et al., 1998; Luke, Hediger, et al., 2003). The general consensus among researchers who have evaluated these twin guidelines is that to qualify as 'enough', total weight gain should be at least 40-45 pounds $(18-20 \mathrm{~kg})$, with an emphasis on adequate weight gain before 24 weeks' gestation (Luke, Brown et al., 1998)

In a recent prospective intervention study, Luke, Brown et al. (2003) observed that pregnancies in women who participated in a specialized program, which included twice-monthly visits, dietary prescription of 3000 to $4000 \mathrm{kcal}$ per day, multimineral supplementation, and patient education, were associated with improved pregnancy outcomes, and lower neonatal morbidity, and consequently reduced cost per twin compared to nonparticipants.

Despite the potential benefit attributed to dietary intervention as an important method of improving

Received 21 November, 2007; accepted 6 December, 2007.

Address for correspondence: Teresinha Simoes MD, Department of Maternal-fetal Medicine and Neonatology, Maternidade Dr. Alfredo da Costa,Lisbon,Portugal.E-mail:teresinhasimoes@netcabo.pt 
the outcome of multiple births, care should be taken in interpretation of the data that led to the recommended weight gains. In particular, it should be noted that in many instances, extrapolations of weight gain, rather than actual weight gain measurements in each period of gestation, were used (Luke et al., 1991; Luke et al., 1993; Luke \& Leurgans, 1996; Luke et al., 1997; Luke, 1998; Luke et al., 1998; Luke, Hediger et al., 2003; Luke, Brown et al., 2003; FlidelRimon et al., 2005). It should also be noted that outcomes may differ according to the method used to estimate weight gain, by the potential confounding effect of prepregnancy maternal weight and body mass index (BMI; Luke, Hediger, et al., 2003; Luke, Brown et al., 2003; Flidel-Rimon et al., 2005; FlidelRimon et al., 2006), as well as by the confounding effect of unrecognized dietary intervention (Luke, Brown et al., 2003), and chorionicity.

In order to circumvent these potential confounders, we used a prospective cohort to analyze the effect of the change in BMI in mothers carrying dichorionic twin gestations in a population that did not receive any dietary intervention. This approach, namely, the change in BMI rather than the change in weight, has not been previously assessed in a prospective cohort.

\section{Material and Methods}

During the period September 1994-March 2006, we followed and delivered 946 twin pregnancies at the Maternidade Dr. Alfredo da Costa, Lisbon, Portugal. This figure represents nearly $1 \%$ of all deliveries in this hospital during the study period. During the study, information about the pregnancy and delivery was first registered on a preset form and then entered into a computerized system. Because a significant proportion of pregnancies were referred at an advanced gestational age, complete data relating to maternal height, pregravid weight, and longitudinal weight measurements were available only for 360 mothers who were followed throughout gestation. In order to avoid the confounding effect of chorionicity, which has a significant effect on fetal growth, we excluded monochorionic twins and focused on 281 dichorionic twin gestations.

Pregnancies were grouped by parity (nulliparas and multiparas) and by body mass index (BMI) using the Centers for Disease Control and Prevention (n.d.; CDC) categories of underweight $(\mathrm{BMI}<18.5)$, normal (BMI 18.5-24.9), and overweight/obese (BMI > 25). We used the CDC categories because they are the most frequently used values in the literature. BMI was calculated from the formula weight $(\mathrm{kg}) /\left(\right.$ height $(\mathrm{m})^{2}$. Following a preliminary assessment of the distribution of BMI, 13 cases of underweight mothers were found and this very small group was also excluded from the analysis.

The following maternal variables were considered in the remaining 268 pregnancies: maternal pregravid weight, maternal height, and maternal weight at each trimester and at birth. Pregravid weight was recorded from referral documentations; maternal height was measured at our service; and finally, maternal weight during each trimester and at birth was recorded from our own measurements (1st trimester 12-14 weeks, 2nd trimester 22-25 weeks, and 3rd trimester 30-34 weeks). When two or more weight measurements were available during a given trimester, an average weight was calculated. All weights were rounded to the first digit.

We derived the BMI-adjusted weight gain, which is defined as the change in BMI between the BMI obtained in a given gestational age expressed as a percentage of the pre-gravid BMI (i.e., the larger the percentage the greater the difference in BMI from a given pre-gravid BMI). This method of presentation of weight gains was used because a given weight gain is expected to have a different meaning for different pregravid BMI. The BMI-adjusted weight gain was calculated for each trimester and then correlated with the total twin birthweight (twin A + twin B) and gestational age at birth. These outcome measures were chosen because they are the most likely to be influenced by maternal weight gain. Finally, we derived means and quartiles of the BMI-adjusted weight gain differences to compare the outcome variables between patients above or below the mean, and between patients in the upper and lower quartile. This was done separately for multiparas and nulliparas because parity, per se, is a powerful determinant of birthweight in twins. (Blickstein, 2005).

The data were evaluated using Microsoft Excel and we used True EPISTAT Software (Math Archives, Round Rock, TX) to perform Student's $t$ tests for continuous variables. We derived $p$ values, and these were considered significant if greater than .05 . The study was approved by the local ethics committee.

\section{$\overline{\text { Results }}$}

A total of 269 mothers (150 nulliparas and 119 multiparas) carrying dichorionic twins were evaluated. The mean maternal age was $30.5 \pm 5.0$ years, the mean height was $162.1 \pm 6.7 \mathrm{~cm}$, the mean pregravid weight was $63.8 \pm 11.4 \mathrm{~kg}$, and the mean pre-gravid BMI was $24.3 \pm 4.3 \mathrm{~kg} / \mathrm{m}^{2}$. By the end of the first trimester, at 12-14 weeks, the average change (\%) in pre-gravid BMI was $7.2 \pm 6.1$, and was similar in nulliparas and multiparas $(7.5 \pm 6.2$ vs. $6.9 \pm 5.9$, respectively). At around mid-gestation, at 22-25 weeks, the average change $(\%)$ in pregravid $\mathrm{BMI}$ was $17.4 \pm 8.2$, and was again similar in nulliparas and multiparas $(18.5 \pm 7.7$ vs. $16.0 \pm 8.6$, respectively). In the third trimester, at 30-34 weeks, the average change (\%) in pregravid BMI was $28.7 \pm 10.8$, and was once again similar in nulliparas and multiparas $(30.2 \pm 9.3$ vs. $26.7 \pm 12.3$, respectively). These average changes in BMI increased in a dose related fashion as pregnancy advanced 
Table 1

Comparison Between Below or Above Average Change in BMI on Total Twin Birthweight and Gestational Age, by Gestational Period and Parity

\begin{tabular}{|c|c|c|c|c|}
\hline & \multicolumn{2}{|c|}{ Nulliparas $(N=150)$} & \multicolumn{2}{|c|}{ Multiparas $(N=119)$} \\
\hline & Total twin birthweight (g) & Gestational age (weeks) & Total twin birthweight $(\mathrm{g})$ & Gestational age (weeks) \\
\hline \multicolumn{5}{|c|}{ Pregravid BMI } \\
\hline$<$ average & $4742 \pm 641$ & $35.8 \pm 1.2$ & $4968 \pm 595$ & $36.1 \pm 1.2$ \\
\hline$>$ average & $4746 \pm 648$ & $35.6 \pm 1.5$ & $5129 \pm 642$ & $36.4 \pm 1.0$ \\
\hline \multicolumn{5}{|c|}{$\Delta \mathrm{BMI}$ at $12-14$ weeks } \\
\hline$<$ average & $4807 \pm 598$ & $35.8 \pm 1.4$ & $5152 \pm 568$ & $36.3 \pm 1.2$ \\
\hline$>$ average & $4650 \pm 697$ & $35.6 \pm 1.4$ & $4861 \pm 637$ & $36.1 \pm 1.1$ \\
\hline \multicolumn{5}{|c|}{$\Delta \mathrm{BMI}$ at $22-25$ weeks } \\
\hline$<$ average & $4792 \pm 624$ & $35.8 \pm 1.4$ & $5039 \pm 590$ & $36.3 \pm 1.1$ \\
\hline$>$ average & $4691 \pm 661$ & $35.7 \pm 1.4$ & $5007 \pm 649$ & $36.1 \pm 1.5$ \\
\hline \multicolumn{5}{|c|}{$\Delta \mathrm{BMI}$ at $30-34$ weeks } \\
\hline$<$ average & $4707 \pm 656$ & $35.5 \pm 1.5$ & $5028 \pm 571$ & $36.3 \pm 1.1$ \\
\hline$>$ average & $4783 \pm 629$ & $35.9 \pm 1.3$ & $5021 \pm 669$ & $36.1 \pm 1.2$ \\
\hline
\end{tabular}

Note: Data are shown as mean $\pm S D$. No significant differences were found.

Table 2

Comparison Between Lower and Upper Quartiles of the Change in BMI on Total Twin Birthweight and Gestational Age, by Gestational Period and Parity

\begin{tabular}{|c|c|c|c|c|}
\hline & \multicolumn{2}{|c|}{ Nulliparas $(N=150)$} & \multicolumn{2}{|c|}{ Multiparas $(N=119)$} \\
\hline & Total twin birthweight $(\mathrm{g})$ & Gestational age (weeks) & Total twin birthweight $(\mathrm{g})$ & Gestational age (weeks) \\
\hline \multicolumn{5}{|c|}{ Pregravid BMI } \\
\hline 1st quartile & $4813 \pm 674$ & $35.9 \pm 1.2$ & $4834 \pm 612$ & $36.2 \pm 1.2$ \\
\hline 4th quartile & $4827 \pm 592$ & $35.7 \pm 1.4$ & $5049 \pm 580$ & $36.3 \pm 1.0$ \\
\hline \multicolumn{5}{|c|}{$\Delta \mathrm{BMI}$ at $12-14$ weeks } \\
\hline 1st quartile & $4772 \pm 636$ & $35.5 \pm 1.5$ & $5153 \pm 440$ & $36.4 \pm 0.8$ \\
\hline 4th quartile & $4716 \pm 718$ & $35.6 \pm 1.4$ & $4908 \pm 636$ & $36.2 \pm 1.2$ \\
\hline \multicolumn{5}{|c|}{$\Delta \mathrm{BMI}$ at $22-25$ weeks } \\
\hline 1st quartile & $4813 \pm 638$ & $35.9 \pm 1.5$ & $5192 \pm 485$ & $36.6 \pm 0.8$ \\
\hline 4th quartile & $4683 \pm 733$ & $35.6 \pm 1.5$ & $5030 \pm 618$ & $36.1 \pm 1.2$ \\
\hline \multicolumn{5}{|c|}{$\Delta \mathrm{BMI}$ at $30-34$ weeks } \\
\hline 1st quartile & $4713 \pm 677$ & $35.6 \pm 1.6$ & $5102 \pm 558$ & $36.5 \pm 0.9$ \\
\hline 4th quartile & $4843 \pm 675$ & $36.2 \pm 1.3$ & $5051 \pm 656$ & $36.2 \pm 1.3$ \\
\hline
\end{tabular}

Note: Data are shown as mean $\pm S D$. No significant differences were found.

\section{$\square 12-14 \quad \square 22-25 \quad \square 30-34$}

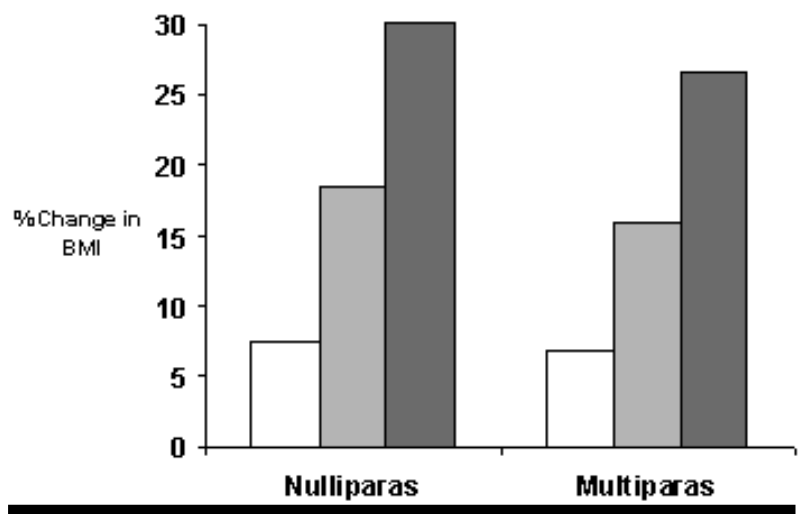

Figure 1

Change in BMI (\%) by gestational period (wks) and parity.
(Figure 1); in addition, changes in BMI were somewhat higher in nulliparas.

The mean gestational age at birth was $36.0 \pm 1.3$ weeks, and the total twin birthweight was $4868 \pm$ $644 \mathrm{~g}$. Table 1 shows the comparison of these outcome measures between maternities below or above the average change in pregravid BMI. The analysis failed to demonstrate an advantage of an above average change in pregravid BMI. Even when the tails of the distributions (i.e., lower vs. upper quartiles) were compared (Table 2), no significant differences were found.

\section{$\overline{\text { Discussion }}$}

Dietary intervention resulting in maternal weight gain is believed to be the only effective prophylactic treatment which improves outcomes in multiple pregnancies (Luke et al., 1991; Luke et al., 1993; Luke 
\& Leurgans, 1996; Luke et al., 1997; Luke, 1998; Luke et al., 1998; Luke, Hediger et al., 2003; Luke, Brown, et al., 2003), although the explanation for this presumable cause-and-effect relationship is still debatable. It seems logical that for adequate growth of more than a single placenta, and for nurture of more than one fetus, additional energy (i.e., caloric) input is required (Blickstein, 2005). However, if this energy is directed to the feto-placental units, maternal weight gain means input of surplus energy. In other words, not only are better outcomes found in cases where food calories are consumed in excess, but also a significant excess of these calories (to produce a significant weight gain) must be present before an effect can be seen. It is therefore plausible in twin pregnancies that maternal weight gain has a significant effect in a priori lean mothers compared to mothers who before pregnancy have an appropriate BMI, or are overweight or obese, as has been previously found in triplet gestations. (Flidel-Rimon et al., 2005; Flidel-Rimon et al., 2006).

It is unclear how a net gain in weight is related to outcomes, because energy is simply stored in the body in the form of glycogen and adipose tissue, and is readily transformed back to energy when needed. This accepted mechanism is directly related to probably different biological implications of a given weight gain for different BMIs. Hence, if weight gain were to affect outcomes, it should be tailored to a given pregravid BMI.

Explanation of the causal relationship between maternal weight gain and improved outcomes is also somewhat hampered by methodological issues. In almost all the studies in question, weight gain is estimated from an average weekly weight gain, which is calculated from maternal weight at a given gestational age and the pre-gravid weight, followed by extrapolation to 24 weeks' gestation. Such extrapolation is necessary because most large registries do not have longitudinal maternal weight assessments (FlidelRimon et al., 2005; Flidel-Rimon et al., 2006), and this method provides the next best estimation. However, the construct of the prospective cohort study conducted by Luke, Brown et al. (2003) could not differentiate between the effects of weight gain per se, and the potentially beneficial effects of close follow up, multimineral supplementation, and patient education (Flidel-Rimon et al., 2006). In addition to these methodological limitations emerging from the need for extrapolation, the unquestionable effects of chorionicity on outcomes are seldom considered in large registries, as chorionicity information is ill-recorded or entirely disregarded. (Flidel-Rimon et al., 2005; Flidel-Rimon et al., 2006). Finally, surprisingly few other studies on the effect of weight gain have been carried out (Kanadys \& Oleszczuk, 2000; Lantz et al., 1996; Rydhstroem \& Walles, 1996; Yokoyama \& Shimizu, 1999).
With these difficulties in mind, we conducted this prospective analysis of the effect of the change in BMI in mothers carrying dichorionic twin gestations in a population that did not receive any dietary intervention. Unlike previous studies, in this cohort we could not find an association between weight gain, in terms of change in BMI, and outcomes, in terms of gestational age at birth and total twin birthweight. Our observations led to different conclusions regarding recommended universal dietary intervention in twin gestations and, therefore, universal recommendations of early weight gain in twins should be confirmed by intention-to-treat, randomized trials.

It should be remembered, however, that we excluded from this study a small number of underweight mothers who might turn out to be the target population for dietary intervention in multiple pregnancies. (Flidel-Rimon et al., 2006). It is thus possible that inclusion of this group of twin mothers would have shown different outcomes (Flidel-Rimon et al., 2005). Additionally, it should be noted that our observations in twins may not apply to triplets, as potential effects of weight gain in higher order multiple pregnancies were not considered in this study.

Finally, the cautious approach that we advocate to the seemingly harmless recommendation of weight gain is best appreciated by the possibility of many normal weight women becoming overweight or even obese by the end of pregnancy, and by the untoward effects of obesity on future health and body image (Flidel-Rimon et al., 2006).

\section{References}

Blickstein, I. (2005). Growth aberration in multiple pregnancy. Obstetrics and Gynecology Clinics of North America, 32, 39-54.

Blickstein, I., \& Keith, L. G. (2005). The decreased rates of triplet births: Temporal trends and biologic speculations. American Journal of Obstetrics and Gynecology, 193, 327-331.

Flidel-Rimon, O., Rhea, D. J., Keith, L. G., Shinwell, E. S., \& Blickstein, I. (2005). Early adequate maternal weight gain is associated with fewer small for gestational age triplets. Journal of Perinatal Medicine, 33, 379-382.

Flidel-Rimon, O., Rhea, D. J., Shinwell, E. S., Keith, L. G., \& Blickstein, I. (2006). Early weight gain does not decrease the incidence of low birth weight and small for gestational age triplets in mothers with normal pre-gestational body mass index. Journal of Perinatal Medicine, 34, 404-408.

Centers for Disease Control and Prevention. (n.d.). Available at http://www.cdc.gov/nccdphp/dnpa/bmi/ adult_bmi

Kanadys, W. M., \& Oleszczuk, J. (2000). [Maternal weight gain during twin pregnancy. Its relationship to the incidence of preterm delivery]. Ginekologia Polska, $71,1355-1359$. 
Lantz, M. E., Chez, R. A., Rodriguez, A., \& Porter, K. B. (1996). Maternal weight gain patterns and birth weight outcome in twin gestation. Obstetrics and Gynecology, 87, 551-556.

Luke, B. (1998). What is the influence of maternal weight gain on the fetal growth of twins? Clinical Obstetrics and Gynecology, 41, 56-64.

Luke, B., Brown, M. B., Misiunas, R., Anderson, E., Nugent, C., van de Ven, C., Burpee, B., \& Gogliotti, S. (2003). Specialized prenatal care and maternal and infant outcomes in twin pregnancy. American Journal of Obstetrics and Gynecology, 189, 934-938.

Luke, B., Hediger, M. L., Nugent, C., Newman, R. B., Mauldin, J. G., Witter, F. R., \& O’Sullivan, M. J. (2003). Body mass index: Specific weight gains associated with optimal birth weights in twin pregnancies. Journal of Reproductive Medicine, 48, 217-224.

Luke, B., Keith, L., Johnson, T. R., \& Keith, D. (1991). Pregravid weight, gestational weight gain and current weight of women delivered of twins. Journal of Perinatal Medicine, 19, 333-340.

Luke, B., \& Leurgans, S. (1996). Maternal weight gains in ideal twin outcomes. Journal of the American Dietetic Association, 96, 178-181.

Luke, B., Gillespie, B., Min, S. J., Avni, M., Witter, F. R., \& O'Sullivan, M. J. (1997). Critical periods of maternal weight gain: Effect on twin birth weight. American Journal of Obstetrics and Gynecology, 177, 1055-1062.

Luke, B., Min, S. J., Gillespie, B., Avni, M., Witter, F. R., Newman, R. B., Mauldin, J. G., Salman, F. A., \&
O'Sullivan, M. J. (1998). The importance of early weight gain in the intrauterine growth and birth weight of twins. American Journal of Obstetrics and Gynecology, 179, 1155-1161.

Luke, B., Minogue, J., Witter, F. R., Keith, L. G., \& Johnson, T. R. (1993). The ideal twin pregnancy: Patterns of weight gain, discordancy, and length of gestation. American Journal of Obstetrics and Gynecology, 169, 588-597.

MacDorman, M. F., Martin, J. A., Mathews, T. J., Hoyert, D. L., \& Ventura, S. J. (2005). Explaining the 2001-2002 infant mortality increase in the United States: Data from the linked birth/infant death data set. International Journal of Health Services, 35, 415-442.

Martin, J. A., Hamilton, B. E., Sutton, P. D., Ventura, S. J., Menacker, F., \& Munson, M. L. (2003). Births: Final data for 2002. National Vital Statistics Reports, 52, 1-113.

Martin, J. A., Hamilton, B. E., Sutton, P. D., Ventura, S. J., Menacker, F., \& Munson, M. L. (2005). Births: Final data for 2003. National Vital Statistics Reports, 54, 1-116.

Rydhstroem, H., \& Walles, B. (1996). Lack of correlation between maternal body weight or weight gain and stillbirth in twin pregnancy. Gynecologic and Obstetric Investigation, 42, 8-12.

Yokoyama, Y., \& Shimizu, T. (1999). [Optimal maternal weight gain in twin and triplet pregnancy]. Nippon Koshu Eisei Zasshi, 46, 604-615. 
Copyright of Twin Research \& Human Genetics is the property of Australian Academic Press and its content may not be copied or emailed to multiple sites or posted to a listserv without the copyright holder's express written permission. However, users may print, download, or email articles for individual use. 